\title{
Impacto de la brecha digital en los procesos de enseñanza y aprendizaje en la escuela de Administración de Empresas de la Escuela Superior Politécnica de Chimborazo
}

\section{Impact of the digital divide in teaching and learning processes in the School of Business Administration of the Polytechnic School of Chimborazo}

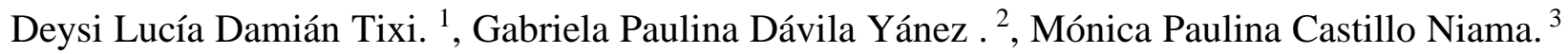
$\&$ Segundo Salvador Cazar Costales. ${ }^{4}$

Recibido: 10-11-2019 / Revisado: 05-12-209 /Aceptado: 26-12-2019/ Publicado: 04-01-2020

\begin{abstract}
.
DOI: https://doi.org/10.33262/cienciadigital.v4i1.1103

The problem of the digital and technological gap is focused on the process, scope and repercussions that are just beginning to show in a changing society, this problem starts a new generation where the digital divide is equivalent to any unequal distribution in access, use or impact that Information and Communication Technologies (ICT) generates among social groups such as actors in higher education (academy, teachers and students), this indicates that considerable groups lack knowledge and skills for the operation of the Internet as well as the computer, and have not noticed the serious problem they face. Therefore, this analysis proposes to carry out a theoretical and qualitative study on the evolution of the digital divide based on generational and technology changes, considering the academic environment in the Escuela de Administration de Empress of the ESPOCH as an object of study through which A research with descriptive scope marks the undeniable rapidity of technological advances and that this has led to events that society has not stopped to examine. However, in the context of higher education the visions of actors such as; The institution of higher education,
\end{abstract}

\footnotetext{
1 Escuela Superior Politécnica de Chimborazo, Facultad de Administración de Empresas, Riobamba, Ecuador, deysi.damian@espoch.edu.ec

2 Escuela Superior Politécnica de Chimborazo, Facultad de Administración de Empresas, Riobamba, Ecuador, gabriela.davila@espoch.edu.ec

${ }^{3}$ Escuela Superior Politécnica de Chimborazo, Facultad de Mecánica, Riobamba, Ecuador, monicap.castillo@espoch.edu.ec

${ }^{4}$ Universidad Nacional de Chimborazo, Facultad de Ingeniería, Riobamba, Ecuador, scazar@unach.edu.ec
} 
teachers and students, will indicate that in some way the impact of the gap by new socialization and communication schemes.

Keywords: Generational evolution, technological evolution, higher education, globalization

\section{Resumen.}

El problema de la brecha digital y tecnológico, se enfoca en proceso, alcances y repercusiones que apenas empiezan a mostrarse en una sociedad cambiante, esta problemática inicia una nueva generación donde la brecha digital equivale a cualquier distribución desigual en el acceso, uso o impacto que las Tecnologías de la Información y la Comunicación (TIC) genera entre grupos sociales como los actores en la educación superior (academia, docentes y estudiantes), esto permite indicar que grupos considerables carecen de conocimiento y habilidades para la operación de la Internet como también del computador, y no se han percatado del grave problema que enfrentan. Por lo tanto, este análisis plantea realizar un estudio teórico y cualitativo sobre la evolución de la brecha digital en función de los cambios generacionales y de la tecnología considerando como objeto de estudio el entorno académico de la Escuela de Administración de Empresas de la ESPOCH en cual por medio de una investigación con alcance descriptivo marca la innegable rapidez de los avances tecnológicos y que esta ha llevado acontecimientos que la sociedad no se ha detenido a examinar. Sin embargo, bajo el contexto de la educación superior las visiones de actores como; la institución de educación superior, docente y alumnos, permitirá indicar que de alguna manera el impacto de la brecha por nuevos esquemas de socialización y comunicación.

Palabras claves: Evolución generacional, evolución tecnológica, educación superior, globalización.

\section{Introducción.}

Los usos de Internet en la vida cotidiana son un buen indicativo de la difusión, las condiciones y la especificidad cultural de la sociedad red en un contexto social (Área \& Manuel, 2019). El Internet es como la vida misma, detrás de cada enlace hay un ser humano, por lo que no se trata de un medio frío y deshumanizado, sino más bien de la intercomunicación de millones de usuarios alrededor de todo el mundo implicando el mayor medio de comunicación en la historia de la humanidad.

La educación, como proceso eminentemente social y determinado por el contexto y las visiones del mundo de sus actores, profesores y alumnos, será de alguna manera modificada por nuevos esquemas de socialización y comunicación; en este trabajo se tiene como objetivo analizar los impactos de la Internet en los estudiantes, y sus posibles repercusiones en los procesos de aprendizaje (Ayala, 2017). 
Por otra parte ante esta situación, cabe plantearse cuál ha sido el efecto de las "Nuevas Tecnologías" en la educación y en el aprendizaje teniendo en cuenta las brechas digitales que a simple vista suelen ser un stock para seguir en el proceso; sin embargo tal parece que el impacto producido ha sido menor que en otros ámbitos y que, en esta ocasión, la educación no ha cumplido con su tradicional papel de palanca de cambio, desde el punto de vista que una reflexión más profunda plantea que lo que hay es un gran retraso debido a las implicaciones de los cambios en la educación, que suponen no sólo invertir en equipamiento y en formación sino en un cambio de actitud o de mentalidad (Gómez, 2019).

En la integración de las tecnologías de la información y las comunicaciones (TIC) en el ámbito de la educación ha ido acompañada por la promesa de que estas nuevas herramientas contribuirían a enfrentar los principales retos que tiene el país en materia educativa. Desde los primeros proyectos de incorporación de tecnologías de la información y las comunicaciones (TIC) en las escuelas a fines de los años ochenta se ha considerado que el uso de estas tecnologías era una prioridad para reducir la brecha digital, mejorar la calidad de los procesos de aprendizaje y aumentar la eficiencia en la gestión institucional y académica (Delgado \& Trujillo, 2014).

En relación a lo descrito la brecha digital es cualquier distribución desigual en el acceso, uso o impacto de las Tecnologías de la Información y la Comunicación (TIC) entre grupos sociales. Sin embargo, estos grupos pueden definirse con base en criterios de género, geográficos o geopolíticos, culturales, o de otro tipo ya que, debido al alto costo de las TIC, su adopción y utilización es muy desigual en todo el mundo (Gómez, 2019).

Esta brecha digital deja a una parte de la sociedad al margen de las nuevas tecnologías es generada principalmente por factores socioeconómicos, geográficos o demográficos y crea una nueva desigualdad que el sistema educativo ha intentado minimizar mediante la alfabetización digital en las universidades y la creación de infraestructura, con el propósito de facilitar el acceso a ellas (Barrio \& Manyoma, 2019).

Aguinaga (2016) destaca que la mayor brecha se encuentra entre los que acceden y los que no acceden a las TIC, y su medida cambia en función del incremento al uso y el consumo es decir, se refiere que más allá del acceso, la brecha digital depende de las habilidades y el aprovechamiento de los usuarios de internet, en concordancia, quien señala que la mayor barrera no es el acceso sino el uso, toda vez que las oportunidades que las nuevas tecnologías crean para un mejor desarrollo profesional de los individuos depende en gran medida de la forma en que las utilizan.

Aunque de manera general la brecha digital se relaciona con el uso de internet, Valera (2015) enuncia otras variables generadoras de exclusión digital con base a un estudio globalizado, donde se refiere que no es de dimensión reducida, lo que la exime de la sujeción 
exclusiva a la población del país; abarca todos los ámbitos de la sociedad y sus efectos se extienden más allá de un territorio determinado.

El término brecha digital describe una brecha en términos de acceso y uso de la tecnología de la información y la comunicación que tradicionalmente se consideraba una cuestión principalmente de acceso ya que en estos tiempos la actualidad, con una penetración global de teléfonos móviles y demás tecnologías que hace que la desigualdad relativa se plantee entre aquellos que tienen más y menos habilidades asociadas.

Ecuador ha sufrido una transformación proactiva y reconocida a nivel mundial en función con la integración de tecnología en los sistemas educativos; siendo de esta manera las políticas públicas se enfocan en el desarrollo de la inclusión social aportando significativamente en la democratización del conocimiento y la reducción de la brecha digital (Gómez, 2019).

Sin embargo, bajo diferentes términos el fenómeno conocido como brecha digital es determinada por la OCDE como el rango o la distancia entre áreas individuales, residenciales, de negocios y geográficas en los diferentes niveles socio-económicos en relación con el libre acceso para introducirse en nuevas tecnologías de la información y la comunicación, también el uso libre de la Internet, esto permitió identificar varias diferencias entre países y entre zonas internas de las mismas; es decir, se puede definir a la distancia existente entre aquellos capaces de usar un ordenador y aquellos que no (Ramos, 2019).

Por otra parte, cambiando los esquemas de la enseñanza y el aprendizaje tradicionalmente existe una brecha generacional entre los profesores y sus estudiantes, generada por las concepciones, paradigmas y visiones propias de cada generación. Sin embargo, en un mundo cambiante, impactado por los rápidos avances de la ciencia y la tecnología, las brechas entre profesores y estudiantes no sólo se limitan a las propias de las generaciones, sino que incluyen diferencias en el uso, manejo y acceso de la tecnología entre otras cosas.

Desde otro punto de vista muchos profesores usan la Internet con cierta frecuencia y quizá hasta utilizan una cuenta de correo electrónico; sin embargo, el uso de la Internet sigue siendo limitado ya que a pesar de la gran utilidad que representan para la investigación ciertos recursos disponibles a través de la Internet, como los bancos de datos y los catálogos bibliográficos, éstos son usados por una porción casi insignificante de profesores, lo cual podría ser ocasionado por la carencia de una política de entrenamiento aprendizaje en función de las necesidades específicas de los usuarios (Selva, 2020).

Existe una brecha digital con relación a los usos de la Internet entre los profesores y sus estudiantes, la brecha se agranda cuando los profesores de mayor edad encuentran menos familiaridad con los usos de la computadora y con Internet; si esto se traslada al aula, parece que la Internet no interviene en el proceso de enseñanza-aprendizaje, así lo consideran los profesores, porque la realidad indica que los estudiantes copian tareas, extrayéndolas literales de la Internet, y recurren 
cada vez menos a las fuentes bibliográficas de las bibliotecas, pues disponen de la Internet como su principal fuente de información

Otra línea de investigación más reciente y muy prometedora es la que se centra en analizar las brechas digitales en los contextos educativos informales. En los últimos años se ha ido consolidando el enfoque de la nueva ecología del aprendizaje (Matsumoto, Aliagas, Morgade, Correro, \& Galera, 2016) que pone de manifiesto que las personas aprendemos muchas cosas y muy relevantes al participar en prácticas sociales como las actividades de ocio, mediadas en gran medida por las TIC. Aunque este tipo de investigaciones son mucho menos frecuentes, se han realizado algunos estudios que muestran que también en estos contextos se aprecian desigualdades tanto en el acceso como en los usos.

Los impactos de la tecnología están creando una nueva realidad social, con cambios inminentes en las instituciones sociales, políticas, económicas y educativas; las relaciones entre los actores también están siendo modificadas (Ramón, 2016). Nos encontramos, pues ante una sociedad distinta, el problema del cambio digital y tecnológico es que se halla en proceso, y sus alcances y repercusiones apenas empiezan a mostrarse; en una sociedad cambiante, surge una nueva generación de estudiantes.

Los estudiantes de hoy en día desde niveles de primaria a universidad representan la primera generación que creció con esta nueva tecnología sin embargo han pasado su vida entera rodeada por el uso de computadoras, videojuegos, reproductores musicales digitales, cámaras de video, teléfonos celulares y todos los demás juguetes y herramientas de la era digital y donde un estudiante promedio o recién graduado de la universidad a lo largo de la vida más o menos cinco mil horas la han pasado leyendo, más de diez mil horas interactuando con videojuegos como también las veinte mil horas viendo televisión, juegos de computadora, correos electrónicos, la Internet, teléfonos celulares y mensajes instantáneos, que son parte integral de sus vidas donde interviene la brecha digital en un ámbito preocupante (Ruiz \& García, 2020).

Se define a los estudiantes de ahora como hablantes nativos del lenguaje digital de las computadoras, de la Internet, a los que denomina nativos digitales. A quienes no nacieron en la era digital y en algún momento, a lo largo de su vida, se han convertido en usuarios de las nuevas tecnologías, los denomina inmigrantes digitales; esta diferenciación con lleva implicaciones importantes para el proceso del aprendizaje; de acuerdo con estos supuestos, las brechas digitales indujeron mucho en el aprendizaje de muchas personas, estudiantes, aunque han transitado y se han adaptado, mantienen sus esquemas iniciales de formación (Alejandro, 2017).

Por el contrario, a los nativos digitales les gustan los procesos paralelos y prefieren realizar multitareas, los gráficos sobre los textos, acceso al azar, funcionan mejor en redes, prosperan en la satisfacción inmediata y las recompensas frecuentes y tienen una marcada preferencia por los 
juegos. Las habilidades de los inmigrantes profesores tienen como características principales las siguientes: proceso lento de información, paso a paso, una cosa a la vez, individualmente y extremadamente formalizado y definitivamente, estas habilidades chocan con las propias de los nativos, a quienes les parecen como fuera de toda realidad (Cabero \& Ruiz, 2017).

Esta desvinculación entre las habilidades de los inmigrantes y las de los nativos digitales, provoca en el proceso de enseñanza-aprendizaje un desfase total, donde los profesores se quejan constantemente de los estudiantes, que aparentemente pierden fácilmente la atención y muestran poco interés por la clase, los materiales y por el profesor. Los estudiantes, por su parte, descubren en los profesores una desvinculación total y poco entendimiento de la realidad, y consideran que los materiales son obsoletos y poco aplicables; encuentran poco sentido por la escuela, y sus habilidades digitales les han permitido realizar sus tareas y trabajos con el mínimo esfuerzo (Zenteno, 2010).

Definitivamente, existen marcadas distancias entre cómo fueron educados los profesores en los temas y tecnologías digitales y la manera en que la tecnología ha impactado a estas nuevas generaciones de los últimos veinte años, creciendo con la tecnología a su lado el problema está ahí, consiste y se hace cada vez mayor, pero ha sido ignorado por otra parte se considera que las instituciones de educación superior y las universidades están a la vanguardia tecnológica porque cuentan con el equipo de cómputo más moderno y una red inalámbrica de Internet, o porque cada salón cuenta con un proyector y una laptop para que el profesor ya que se están haciendo cambios de forma, pero no de fondo (García, 2017). La realidad es que los profesores no cuentan con las habilidades pedagógicas y tecnológicas para apropiarse de nuevas metodologías de la enseñanza y adaptarse a los nuevos estudiantes. Solamente se pueden considerar dos opciones: esperar a que los nativos digitales sean algún día profesores, o que los inmigrantes digitales empiecen a asumir que se requiere cambiar y adaptarse a una nueva era.

Otros estudios determinan que ciertas iniciativas responden a puntos de vistas optimistas enfocados en el impacto de las tecnologías de la información y la comunicación, como también en el desarrollo en consecuencia la erradicación de la problemática del problema de la Brecha Digital se realizará por medio de la introducción de computadores personales con acceso a Internet considerando el debido acompañamiento y guía para el uso de las mismas. Sin embargo, el diseño de contenidos culturalmente adecuados a cada contexto es olvidados, así mismo, las comunidades capacitadas en determinados procesos de instrucción y el equipamiento tecnológico no son consideradas importantes, lo que afecta significativamente la sustentabilidad a largo plazo de proyectos de esta naturaleza (Robles, 2017).

Cuando hablamos de acceso a la tecnología, no sólo nos referimos a contar con tecnología, sino a la existencia de una brecha mental, que comprende la habilidad en el manejo de computadoras, la capacidad en el idioma inglés que ha sido bautizado como el idioma de la red, porque $75 \%$ de la información generada en la Internet es en inglés y sentirse cómodo y familiarizado con estas 
tecnologías y su aprendizaje o comunicación; en ese sentido, experiencias recientes han demostrado que instalaciones costosas de hardware de computadoras pueden ser completamente inútiles si nadie sabe cómo utilizarlo y mantenerlo (Casamayou \& González, 2017).

Mientras las universidades se han preocupado por contar con la mejor y más actual tecnología de punta, el desarrollo de capacidades y de nuevas habilidades acordes a la incorporación tecnológica ha sido subordinado $\mathrm{y}$, en muchos casos, totalmente olvidado; el determinismo tecnológico violenta la idea de que la tecnología constituye un apoyo para la educación, convirtiéndose justamente en lo contrario (brecha digital) (Zenteno, 2010). En muchos casos, las plataformas tecnológicas empleadas en los procesos de enseñanza, se ve como el punto de llegada y no como el punto de partida hacia la creación de nuevos espacios de aprendizaje y poder tener una buena visualización de comunicación y aprendizaje tecnológico.

La búsqueda de la información en la infraestructura tecnológica deja en un segundo plano el interés en los contenidos educativos es decir la brecha digital interviene muchísimo en ello, con lo cual relegan la construcción del conocimiento bueno en este contexto, es importante destacar la importancia de las universidades en la distribución social del conocimiento científico y socialmente legitimado; mientras las universidades y las instituciones de educación superior ni siquiera han reconocido el problema de las brechas digitales, los estudiantes universitarios pertenecen a una generación en la cual la tecnología y la digitalización son parte de su vida personal y social; con ella han crecido y formado una nueva identidad, una nueva forma de relacionarse y de comunicarse, han desarrollado nuevas habilidades, distintas a las de aquellos a quienes la tecnología alcanzó en etapas de madurez y desarrollo avanzadas (Reimers, 2019).

Por otra parte la integración de las tecnologías digitales en instituciones de educación como la educación superior (universidades), los docentes presentan un bajo nivel de competencia digital, en congruencia con la situación problémico digital global sin embargo, no es un proceso sencillo la revisión de los obstáculos o barreras que se generan procesos de integración de las TICS en un sistema educativo son las principales barreras de hacer progresar o conseguir los objetivos propuestos (KEIL, 2015). En otros estudios se puede identificar entre barreras internas factores como: creencias, prácticas y resistencia, externas, acceso, tiempo, apoyo, recursos. Por otra parte, otros estudios se enfocan en las dificultades a nivel de escuela donde interviene la falta de eficacia de la formación, recursos, accesos entre otros mientras en los docentes interviene la falta de tiempo, de confianza, resistencias al cambio.

La actual incorporación desigual de las TIC en la sociedad ha provocado diferencias en las oportunidades de desarrollo entre grupos de poblaciones, al establecer una distancia entre las que tienen acceso a ellas y las que no (brecha digital) (2016). De acuerdo con la UNESCO en el año 2005, la brecha digital es la distancia o la separación existente entre los individuos que utilizan las TIC como parte rutinaria de su vida diaria y las que no tienen acceso a ellas, o bien, no saben cómo usarlas. 
En las universidades el aprendizaje implica de modo necesario un cambio en el paradigma, donde se requiere pasar de un modelo educativo centrado en la enseñanza a otro que gire en torno al aprendizaje y esto es aún más evidente cuando se integra internet en los procesos educativos como nuevo medio para acceder a la multiplicidad de fuentes de información ubicadas en la red global. Según Sigalés \& Mominó (2015) Internet es, después de la televisión, el medio de comunicación de mayor influencia en niños y jóvenes y es una excelente herramienta de comunicación, educativa y productiva que permite a niños y jóvenes aprender, investigar, buscar información o comunicarse con familiares o amigos.

Al mismo tiempo se tiene como objeto de estudio el uso educativo de las tecnologías en la educación es decir en este sentido, muchos ven en las TIC una avenida para liberar la educación formal de los confines del aula y no sólo como una oportunidad para desarrollar una educación más personalizada que responda a las características e intereses de cada alumno si no también tener en cuenta que en la actualidad se está combatiendo en universidades específicamente en el ámbito educativo sobre las brechas digitales (Somekh, 2017).

Sin embargo, la lenta adopción y el uso limitado de las nuevas TIC en la mayoría de los salones de clase alrededor del mundo es un ejemplo prototípico de la dificultad y complejidad de la introducción de cambios fundamentales en los sistemas educativos.

La integración de la tecnología a la enseñanza se describe en todos ellos como un proceso complejo para la mejora de aprendizaje, en el que el maestro, las necesidades de acceso y capacitación, la cultura escolar y la percepción del docente son centrales y de acuerdo con lo escrito la capacitación sobre las brechas al docente es importante, pero si no está vinculada al aprendizaje colectivo, no puede influir a la cultura de la universidad.

En los últimos dos años, se ha iniciado un programa uno de ellos es Digital Educación, este programa se enfoca en acortar la gran brecha digital existente en determinada población donde las estadísticas describen índices bajos en el desconocimiento del uso de las tecnologías, sin embargo, este programa considera una transformación de los centros de estudios por medio de una dinámica de infraestructura tecnológica de dotación de computadores a equipos de gestión, docentes y estudiantes. La instalación de pizarras digitales en aulas y de servidores en los centros educativos y de formación docente para la integración de las tecnologías digitales, en consecuencia, la investigación hace reflexionar lay presenta resultados en relación al componente formativo del proyecto Digital Educación (Tondeur, Braak, \& Valcke, 2016).

La implementación de cualquier innovación dentro de un sistema educativo necesita de la adopción de una metodología que planifique los cambios ya que en este proceso participan los diferentes estamentos implicados en el proceso educativo (Marqués, 2016). Por lo tanto, la propuesta ante dicha problemática se presenta un modelo de planificación de las tecnologías enfocada en la necesidad a nivel de distrito, y estos a la vez analizan las posibilidades y deficiencias de tecnologías, como de recursos y procesos formativos, para luego descender al nivel de escuela. 
Passey (2006) describe que en las instituciones educativas desarrollan la implantación de las tecnologías por medio de obras de acondicionamiento mejorando el nivel de calidad de las infraestructuras tecnológicas en las escuelas y universidades dejando así bien claro que también hay que tener en cuenta el problema de seguridad y de mantenimiento; ya que estos aspectos han sido importantes en el programa Digital Educación. Estos programas cuando se implementan la política de implantación de las tecnologías en las instituciones de educación en imperante contar con los siguientes elementos:

- Equidad: en el proceso de la implementación de las tecnologías, es necesario considerar en la decisión enfocadas en las prioridades a atender.

- Participación: la innovación tecnológica y la implantación de las misma requiere de la colaboración de todos quienes participan en los procesos académicos y sociales que de alguna manera van a ser afectados por su implementación esto incluye no solo a docentes, directores de escuelas, de regionales y distritos, también a los padres, comunidades, sindicatos, sociedad civil y sector privado.

- Transparencia: en cuanto a la inversión de recursos tecnológicos la implantación de las tecnologías en las escuelas representa una de las decisiones que se adopten en relación con las infraestructuras (tipos y modelos de dispositivos a comprar), como los procesos de formación y apoyo, posean requerimientos en cuanto a la trazabilidad para mejorar la evaluación de su impacto en el sistema educativo.

- Conectividad: No se trata sólo de dotar de conectividad a las escuelas, sino al sistema mediante o a través de la inclusión de las tecnologías en las escuelas y aulas, se requiere disponer de una red de conexión.

- Monitorización y evaluación: El proceso de implantación de tecnología debería contemplar el proceso de monitorización y evaluación de resultados del proyecto a través del establecimiento de indicadores claros.

- Evaluación y diseño de software: La implementación de un sistema o programa educativo con base en las tecnologías tiene como requerimiento la realización de una evaluación del software a utilizar, de esta forma se espera potenciar a la medida de lo posible las opciones de "open source".

- Determinación del hardware: para el desarrollo del programa existe también una disyuntiva ¿computadores o tabletas? Esta decisión es relevante ya que va de la mano con la relación de la integración de las tecnologías en las escuelas o universidades que tienen que ver con qué tipo de dispositivo se selecciona para acceder a los recursos digitales como patrones o tendencia, tal como se presenta.

Producción de contenidos digitales: los contenidos son representados por los contenidos digitales, multimedia, interactivos estos productos representan un rol determinante debido a que los contenidos son un valor añadido, el problema se enfoca en que los docentes difícilmente van a utilizarlos en sus aulas. Por ello, un plan de implantación de tecnologías en los entornos educativos, las universidades necesitan de une espacio adecuado donde la estrategia de reutilización, generación, difusión, distribución, selección, producción de contenidos digitales abastezca a todos los niveles del sistema educativo.

Pero la integración de las tecnologías en instituciones educativas donde los docentes presentan un bajo nivel de competencia digital, eso implica replantearse ¿cuáles son los factores que inciden en 
el desarrollo eficiente o que dificultan la adopción de las tecnologías en especial en los países en vías de desarrollo y el cual la brecha digital está surgiendo fuerte? en un reciente artículo, Clayton Wright se decantaba por cinco factores condicionantes claves: la energía electricidad necesaria para el funcionamiento y mantenimiento adecuado de los computadores; conectividad, acceso a internet de banda ancha, con o sin cable; la actualización en cuanto a la formación de los profesores en el uso de tecnologías; incorporación de la valoración de la docencia (salario, condiciones de trabajo, calidad de formación inicial, innovatividad); sostenibilidad de la innovación con el mantenimiento de proyectos y de infraestructuras (López, 2017).

La segunda aprender con tecnologías para enseñar con tecnologías a nivel global causa de rechazo donde el cuerpo docente no usa las tecnologías, esto implica una falta de actualización en la parte formativa del profesional, sin embargo, también está relacionada la falta de confianza en sí mismo cuando utilizan tecnologías (2017).

En los casos estudiados fueron encontrados los siguientes obstáculos: acceso limitado, software inadecuado y preocupaciones éticas y brecha digital, sin embargo, se solicita repetidamente capacitación en la integración de las TIC; no en su manejo ya que los estudios mencionados identifican mejoras directas e indirectas en los logros de los estudiantes y algunos coinciden en la relevancia de la cultura escolar y los maestros para la innovación educativa. También convergen los estudios de caso citados en algunos de los problemas de cambio en el proceso enseñanza aprendizaje, sobre todo la centralidad del maestro como protagonista del cambio mismo y la trascendencia del contexto donde se lleva a cabo la innovación; se identificó la falta de acoplamiento entre política y práctica educativas aparentemente, las tecnologías más fáciles y flexibles tienen mejores posibilidades de integración, pues son capaces de ajustarse a las necesidades, capacidades y expectativas locales también se requieren investigaciones acerca de las mejores prácticas con TIC (Zenteno, 2010).

\section{Metodología}

El siguiente estudio se ha realizado en la Escuela Superior Politécnica de Chimborazo, con alumnos de escuela de Administración de Empresas, estudiantes de tercer semestre paralelos: "1" “2” y “3” durante el periodo académico Marzo - Julio 2019, para llevar a cabo nuestro estudio nos hemos valido de un enfoque cualitativo de investigación que nos permitió realizar inferencias sobre un objeto que hasta el momento no ha sido sometido a investigación en profundidad y el programa de digital educación fue escogido para desarrollar nuestro estudio es el análisis de contenido, el cual constituye un conjunto de técnicas de análisis de comunicaciones tendiente a obtener indicadores cuantitativos o no; por procedimientos sistemáticos y objetivos de descripción del contenido de los mensajes, permitiendo la inferencia de conocimientos relativos a las condiciones de producción, recepción. 
Este diseño se escogió para investigar otros materiales y documentos de la disciplina en cuestión y nos ha parecido particularmente útil, dado que, como señalan sus referentes, favorece la formulación de inferencias e hipótesis en torno a materiales cuya indagación no ha sido sistemática.

Actualmente se trata de un cambio donde los tres aspectos principales, la brecha global que se presenta entre distintos países, la brecha social es la que incide a nivel interno de un país y la brecha democrática que se enfoca entre quienes participan y quienes no participan de los asuntos públicos en línea (Andión, 2017).

Otra corriente de investigación centra aspectos cuantitativos de la brecha digital, identificando diferencias a nivel estadístico para el acceso a las tecnologías de la información y la comunicación, según un extenso abanico de factores socios demográficos entre las que destacan el sexo, la edad, el nivel de ingresos, la escolaridad, la raza y el lugar de residencia.

Uno de los aspectos más recientes que han sido analizados sobre la brecha digital, tiene que ver no solamente con el acceso a Internet, sino con la calidad de dicho acceso y la disponibilidad de conexiones de banda ancha que permitan acceder a contenidos multimedia en tiempos y costos adecuados al contexto de los usuarios.

De estas forma específica, la investigadora neerlandesa José van Dijck identifica cuatro dimensiones en el acceso: la motivación para acceder, el acceso material, las competencias para el acceso, y el acceso para usos avanzados o más sofisticados y plantea que la brecha digital está en constante evolución, dado el surgimiento de nuevos usos tecnológicos, que son apropiados más rápidamente por aquellos que tienen el acceso en forma más permanente y de mejor calidad, determinado por dicho ancho de banda (2015).

\section{Resultados}

Se ha asumido un modelo de formación centrado para la Escuela Superior Politécnica de Chimborazo en la Escuela de Administración de Empresas perteneciente a la Facultad de Administración de Empresas ya que se ha pretendido huir de modelos de formación masiva y tecno céntrica, para el desarrollo y conocimiento de las nuevas tecnologías y la evolución de brecha digital para enfocarse en un esquema cuyos principios han sido los siguientes:

- Aprendizaje expandido: En la Escuela Superior Politécnica de Chimborazo en la Escuela de Administración de Empresas se desarrolla acciones que en diferentes momentos y contextos también se visualiza los requerimientos de circunstancias o situaciones presenciales y no presenciales con el aporte de dispositivos tecnológicos y de aprendizaje también. La mezcla entre presencialidad y presencial no es fija, esta se adapta en función del tipo de contenido a desarrollar y de la capacidad de los actores de acceder a las 
situaciones de formación en función de la disponibilidad de desplazamientos o de conectividad.

- Aprendizaje enriquecido: las diferentes acciones alineadas a la formación aportan a los diferentes actores con una amplia variedad de recursos tanto humanos como digitales donde a los estudiantes de los tres cursos de diferentes paralelos pertenecientes al tercer semestre de la Escuela de Administración de Empresas puedan cumplir con requerimientos de las buenas prácticas bien documentadas relacionadas con los contenidos y competencias a aprender.

- Aprendizaje situado: en cuanto a la formación es necesario considerar que las acciones deben estar basadas en factores que involucran el entorno académico y de cada uno de los actores que participa; así la Escuela Superior Politécnica de Chimborazo en la Escuela de Administración de Empresas tratara de que las acciones de formación incluyen actividades que facilitan la puesta en práctica o transferencia de aprendizaje de los contenidos en las situaciones concretas en las que los actores se desempeñan en la brecha digital dentro de las TICs. Esta es una variable condicional e imprescindible en el diseño e implementación de toda acción formativa, para ello, los planes de mejora que son elaborados por los centros educativos serán el componente estructural de la formación continua por su continua evolución.

- Aprendizaje activo: Como consecuencia de lo anteriormente mencionado, toda acción de formación de la Escuela de Administración de Empresas requiere a los diferentes cursos como actores que movilizan conocimientos y por medio de las competencias desarrollan actividades formativas en las que realizan variedad de tareas basadas en la comprensión, diálogo, construcción de conocimiento, aplicación, indagación, producción o evaluación e investigación actualizada.

- Aprendizaje colaborativo: Los estudiantes tendrán formación donde incluye espacios formales o informales, presenciales o virtuales, a través de los cuales los diferentes actores deberán colaborar con otros actores próximos o lejanos, presenciales o virtuales para el desarrollo de actividades formativas específicas de cada acción de formación. De esta manera uno de los objetivos principales de la Escuela de Administración de Empresas es que toda acción de formación sea la constitución de comunidades de práctica en torno a las diferentes temáticas y preocupaciones relevantes para la mejor educación.

- Aprendizaje mediado: las diferentes acciones que se desarrollan en el entorno académico el cual se implementa la tecnología para la integración de los actores se debe de considerar factores que incluye la mediación o acompañamiento presencial y virtual, los estudiantes tendrá que por medio de los facilitadores con formación y buenas prácticas en el área específica del contenido de formación desarrollen por medio de trabajos e investigaciones una continua mejora, se realizará todo o mencionado con el objetivo de mejorar su aprendizaje.

- Aprendizaje flexible: en la ESPOCH la Escuela de Administración de Empresas debe de considerar los requerimientos en cuanto a la participación por parte de los diferentes cursos y con sus respectivos estudiantes en actividades de formación considerada obligatoria, pero también con la posibilidad de que sean electivas, en función de los intereses y motivaciones de los sujetos implicados.

- Aprendizaje dirigido: Toda acción de formación tiene presente la necesidad de mejorar los aprendizajes de los estudiantes de los diferentes paralelos y semestres de la Escuela de Administración de Empresas, por lo tanto, al establecer condiciones que permitan por 
medio de la formación de los actores generen un impacto directo en la calidad de los aprendizajes de los estudiantes en las aulas cumpliendo con sus respectivas funciones.

- Las funciones asignadas a los facilitadores han sido:

- Realizan un diagnóstico de necesidades formativas en relación con las competencias digitales para cada una de sus escuelas bajo su propia responsabilidad.

- Establecen una relación tanto constructiva como de confianza con el equipo directivo y el profesorado del centro educativo al que pertenecen.

- Diseña, en consenso con el profesorado el Plan de Formación de la escuela para la mejora de las competencias digitales y su integración en la enseñanza logrando una educación significativa.

- Desarrolla acciones de formación en variadas modalidades (semanarios, talleres, capacitaciones, cursos, etc.), dirigidas al profesorado y al equipo directivo en relación con las competencias digitales tanto en su integración como en la dirección y la enseñanza.

- Realiza observaciones de aula por parte de docentes apoyando el uso de las tecnologías durante todo el proceso de enseñanza.

- Realiza demostraciones en las aulas de un uso eficiente de las tecnologías digitales en el área curricular correspondiente a su especialidad.

- Cumple el calendario de visitas y actividades con cada uno de los centros docentes asignados.

- Cumplimenta los impresos de seguimiento online para cada una de las actividades desarrolladas en cada escuela.

- Responde en tiempo y forma a los requerimientos de valoración e informes solicitados por la coordinación del programa.

- Informa en el tiempo establecido al Coordinador Distrital de Facilitadores en relación con las actividades desarrolladas hasta el momento.

- Apoya al profesorado en el diseño de actividades y evaluación de los aprendizajes de los alumnos utilizando tecnologías digitales.

- Tomando en cuenta lo descrito la Escuela Superior Politécnica de Chimborazo en la Escuela de Administración de Empresas; usaron por primera vez componentes que ayudaron al aprendizaje en un modelo multifacético de acceso a Internet y a la evolución de brecha digital, los resultados de la investigación ratifican la multidimensionalidad del alto nivel de abstracción de los conceptos de segundo orden utilizados. Es decir, el constructor de segundo orden: acceso material a Internet, las dimensiones acceso material y satisfacción con el acceso mostraron una incidencia significativa y positiva, con un mayor impacto de la primera dimensión mencionada.

\section{Conclusiones.}

- La complejidad de lo investigado de la brecha digital y sus diferentes aproximaciones imponen una exploración epistemológica continua de los supuestos que pretenden participar en la construcción de sus diferentes teorías, en el marco de este esfuerzo, el presente estudio validó satisfactoriamente el uso de componentes de orden superior en la modelación multietápica de la brecha digital y la evolución de la técnica de aprendizaje a lo lago de todos los años. 
- Los resultados confirman que la multidimensionalidad y el alto nivel de abstracción de los constructos de segundo orden como son: el acceso material a Internet y acceso al uso provechoso de la red en consideración a dichos constructos fueron fortalecidos ocupando nuevas dimensiones como la satisfacción del estudiante y la experiencia de uso, que permitieron lograr un oportuno planteamiento nomológico del modelo de investigación. De esta manera el estudio, la disponibilidad del acceso material a Internet y la satisfacción con el servicio resultaron de alto impacto en la accesibilidad; mientras que el uso dentro de la escuela fue altamente aprovechado en el uso de la red.

- Tomando en cuenta lo descrito el modelo multifacético de acceso a Internet fue propuesto para el análisis de la accesibilidad y el aprovechamiento de Internet de la población en general, su aplicación en el contexto de usuarios frecuentes en el país y en este caso la Escuela Superior Politécnica de Chimborazo mostró también una validez significativa de todas las hipótesis planteadas al inicio de esta investigación.

- De esta manera, el uso frecuente de Internet no parece traducirse en todos los casos en habilidades de uso y aprovechamiento de la red; estos aspectos parecen variar en función de las características socioeconómicas de los usuarios y las plataformas tecnológicas elegidas para acceder a la red.

- Desde la perspectiva del tamaño de las localidades donde habitan los usuarios frecuentes, se encontraron diferencias significativas de la incidencia del acceso material a Internet en las habilidades de acceso a la red, entre los tres cursos de los diferentes paralelos de la Escuela de Administración de Empresas. Estas diferencias parecen reflejar más bien a las variaciones estructurales que caracterizan a los estudiantes.

- De la misma manera, se revelaron también otras diferencias entre grupos de tamaño de localidades, que parecen fundamentadas principalmente por los niveles de integración y el posicionamiento orgánico inherentes a los sistemas dentro de la escuela; en este sentido, las diferencias detectadas entre el acceso material a Internet y el acceso al uso provechoso de la red, por un lado, y entre las habilidades de acceso y el acceso al uso provechoso, por el otro, son ilustrativas del impacto sistémico que atraviesa la trama territorial más allá de la variación del tamaño poblacional y también global.

- Es importante subrayar la aparición, en los hallazgos de la presente investigación, de un diseño educación para la accesibilidad en las localidades y no solo podría aplicar en dichas si no también en la Escuela Superior Politécnica de Chimborazo, y otro para la incidencia de las habilidades en el uso provechoso de Internet en las localidades.

- En conjunto, estos resultados parecen indicar la necesidad de continuar la investigación de la apropiación final de Internet, poniendo, a futuro, una atención especial en los diferentes contextos de integración territorial.

\section{Referencias bibliográficas.}

Aguinaga, J. (2016). El uso de las TIC, su influencia en los cambios individuales y sociales. Jóvenes e identidades.

Alejandro, P. (2017). Nativos e inmigrantes digitales: ¿brecha generacional, brecha cognitiva, o las dos juntas y más aún. Revista mexicana de Investigación Educativa, enero marzo.

Andión, M. (2017). Criterios y recomendaciones para la apropiación de las TIC en las escuelas públicas. Equidad tecnológica en la educación básica. 
Área, \& Manuel. (2019). Sociedad de la información y analfabetismo tecnológico. Revista Diálogos.

Arenas, M. (2015). Brecha digital de género: la mujer y las nuevas tecnologías. Anuario Facultad de Derecho-Universidad de Alcalá.

Ayala, A. (2017). La brecha digital continúa reduciéndose.

Barrio, M., \& Manyoma, E. (2019). Mujer y Educación Superior en el Caribe Colombiano. En REOALCEI, La investigación universitaria en red en América Latina y sus diferentes, 4477.

Cabero, J., \& Ruiz, J. (2017). Las Tecnologías de la Información y Comunicación para la inclusión: reformulando la brecha digital. International Journal of Educational Research and Innovation, 16-30.

Casamayou, A., \& González, M. (2017). Personas mayores y tecnologías digitales: desafíos de un binomio. Psicología, Conocimiento y Sociedad, 152-172.

Delgado, M., \& Trujillo, J. (2014). Los equipos directivos de educación primaria ante la integración de las Tic. Revista De Medios Y Comunicación.

García, Á. (2017). Alfabetización digital. Razón y Palabra, 66-81.

Gómez, D. (2019). Brechas y asimetrías que emergen en la era digital. Revista Electrónica de Investigación Educativa.

Gómez, D. (2019). Una aproximación a la evolución de la brecha digital entre la población joven. Revista Española de Sociología.

KEIL, M. (2015). Comparing Continued Use Behavioral Models of the Socio-economically Advantaged. Understanding Digital Inequality.

López, G. (2017). Alternativas metodológicas en educación superior: nuevas tecnologías de información y comunicación en la difusión de la investigación. Ciencias Sociales y Humanidades.

Marqués, P. (2016). Funciones y limitaciones de las TIC en educación La revolución educativa en la era de internet. Impacto de las TIC en el mundo educativo.

Matsumoto, M., Aliagas, C., Morgade, M., Correro, C., \& Galera, N. (2016). Young children and digital technology. A qualitative exploratory study.

Passey, D. (2006). analyzing uses of information and communication technologies by primary and secondary school pupils with learning frameworks. Technology enhancing learning.

Ramón, S. (2016). Brecha digital en procesos de aprendizaje y educación. Educativa.

Ramos, J. (2019). Percepción del profesorado universitario sobre la integración de las Tecnologías de la Información y la Comunicación (TIC) en las modalidades docentes. Influencia del género y la edad, 128-146.

Reimers, F. (2019). Diálogo informado: el uso de la investigación para conformar la política educativa, Distrito Federal. Psicología, Conocimiento y Sociedad.

Rensky, M. (2016). Nativos digitales, inmigrantes digitales. On the Horizon, 9.

Robles, J. (2017). Por qué la brecha digital es un problema social. Por qué la brecha digital es un problema social. 
Vol. 4, N¹, p. 304-320, enero - marzo, 2020

Ruiz, C., \& García, G. (2020). Aprendizaje-Servicio en escenarios digitales de aprendizaje: propuesta innovadora en la educación superior. Revista Iberoamericana de Educación a Distancia, 183-198.

Sánchez, L., Reyes, A., \& Ortiz, D. (2017). El rol de la infraestructura tecnológica en relación con la brecha digital y la alfabetización digital en 100 instituciones educativas. Calidad en la educación.

Selva, A. d. (2020). Escenarios y desafíos de la ciudadanía digital. Revista Mexicana de Ciencias Políticas y Sociales.

Sigalés, C., \& Mominó, J. (2015). TIC e innovación en la educación escolar española: estado y perspectivas. Editorial Telos.

Somekh, B. (2017). Pedagogy and learning with ICT. Researching the art of innovation.

Tondeur, J., Braak, J., \& Valcke, M. (2016). Towards a typology of computer use in primary education. Journal of Computer Assisted Learning.

Varela, J. (2015). La brecha digital en España. Estudio sobre la desigualdad postergada.

Zenteno, A. (2010). Las TIC en la educación media superior. Propuesta de tesis doctoral, Monterrey, México: Tecnológico de Monterrey. 
Vol. 4, $\mathrm{N}^{\circ} 1$, p. 304-320, enero - marzo, 2020

\section{PARA CITAR EL ARTÍCULO INDEXADO.}

Damián Tixi, D. L., Dávila Yánez , G. P., Castillo Niama, M. P., \& Cazar Costales, S. S. (2020). Impacto de la brecha digital en los procesos de enseñanza y aprendizaje en la escuela de Administración de Empresas de la Escuela Superior Politécnica de Chimborazo. Ciencia Digital, 4(1), 303-320. https://doi.org/10.33262/cienciadigital.v4i1.1103

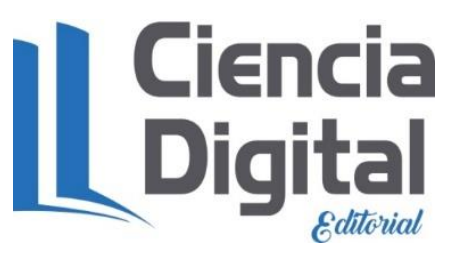

El artículo que se publica es de exclusiva responsabilidad de los autores y no necesariamente reflejan el pensamiento de la Revista Ciencia Digital.

El artículo queda en propiedad de la revista y, por tanto, su publicación parcial y/o total en otro medio tiene que ser autorizado por el director de la Revista Ciencia Digital.
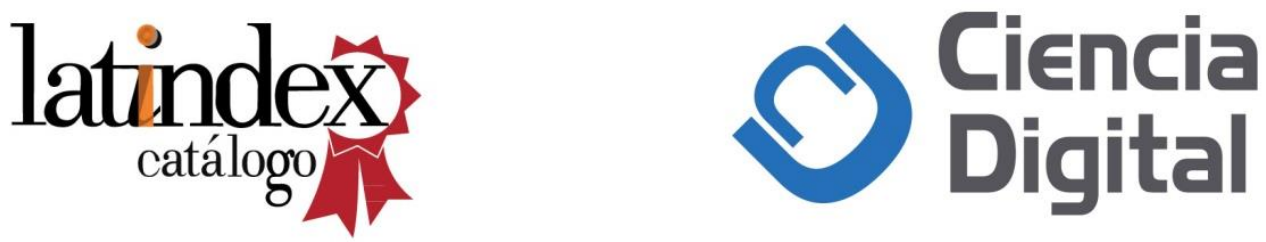